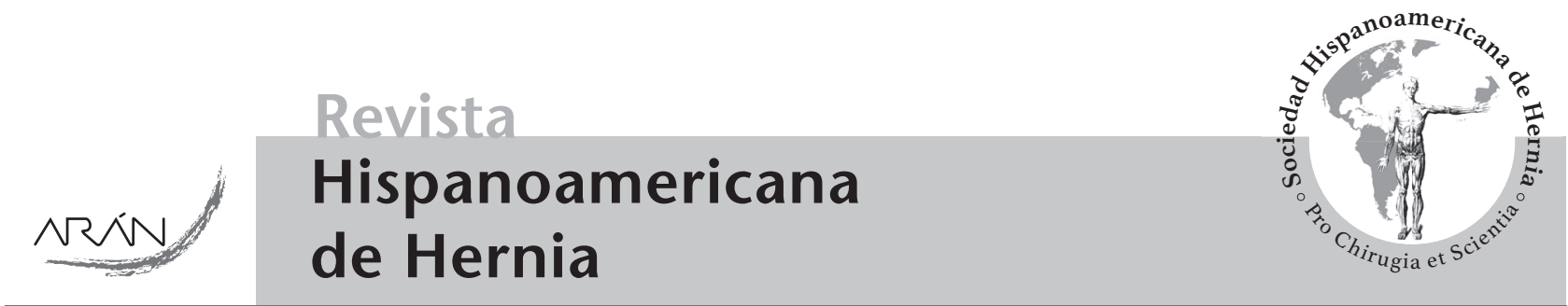

Nota clínica

\title{
Tratamiento de la hernia inguinoescrotal gigante con pérdida de domicilio: un desafío quirúrgico
}

\section{Treatment of giant inguinoscrotall hernia with loss of domain: a surgical challenge}

\section{Fernando Luis Begliardo, Pablo María Arias, Milagros Corpacci, Paula Daniela Albornoz, Aldo Fabián Lerda}

Servicio de Cirugía General. Nuevo Hospital San Roque. Córdoba (República de Argentina)

Recibido: 29-08-2017

Aceptado: $31-10-2017$

\section{Palabras clave:}

Hernia inguinoescrotal,

neumoperitoneo preoperatorio, hernioplastia.

\section{Resumen}

Introducción: Se definen como hernias inguinoescrotales gigantes a aquellas que se extienden por debajo del punto medio de la cara interna del muslo, con el paciente en posición de pie. Debido a la pérdida de domicilio que se puede presentar en estos casos, durante la cirugía, al reintroducir el contenido herniario a la cavidad abdominal, se puede producir un síndrome compartimental abdominal.

Caso clínico: Se describe un caso de un hombre de 50 años de edad con una hernia inguinoescrotal de 15 años de evolución, con contenido intestinal y omental. Se le realizó neumoperitoneo progresivo preoperatorio. La hernioplastia fue llevada a cabo con la colocación de una malla de polipropileno libre de tensión. El paciente tuvo una recuperación sin complicaciones ni recurrencias.

Discusión: El manejo quirúrgico de las hernias inguinoescrotales gigantes que han perdido el domicilio, es un desafío para el cirujano. A lo largo de la historia se han descrito diversas técnicas para llevar a cabo su reparación. Entre ellas, en 1940, Goñi Moreno introduce el concepto de neumoperitoneo progresivo preoperatorio. Es importante conocer los beneficios y riesgos de cada una y saber aplicarlas acorde a cada paciente.

El neumoperitoneo progresivo es una opción válida para reparación de las hernias inguinoescrotales gigantes. Permite, previo a la cirugía, un aumento del volumen de la cavidad abdominal y una disección neumática del saco herniario y de su contenido.
Key words:

Inguinoscrotal hernia, preoperative pneumoperitoneum, hernioplasty.

\begin{abstract}
Introduction: Giant inguinoscrotal hernia has been defined as those that extend below the midpoint of the inner thigh with the patient in standing position. Due to the loss of domain that may occur in these hernias, during surgery an attempt to reintroduce the hernia's content into the abdominal cavity, can lead to an abdominal compartment syndrome.

Case report: We describe a case of a 50-year-old with an inguinoscrotal hernia with 15 years of evolution, with intestinal and omental content. He underwent preoperative progressive pneumoperitoneum. The hernia repair was carried out by placing a mesh tension-free polypropylene. The patient had an uneventful recovery or recurrence.

Discussion: The surgical management of giant inguinoscrotal hernias with loss of domain is a challenge for the surgeon. Throughout history, various techniques have been described to carry out repairs to carry out repairs. Among them, in 1940, Goñi Moreno introduces the concept of progressive pneumoperitoneum preoperative. It is important to know the benefits and risks of each and know how to apply according to each patient.

Progressive pneumoperitoneum is a valid option for repairing giant inguinoscrotal hernias. Prior to surgery, it allows an increase in the volume of the abdominal cavity and a pneumatic dissection of the hernia sac and its contents.
\end{abstract}

* Autor para correspondencia. Fernando Luis Begliardo. Servicio de Cirugía General. Nuevo Hospital San Roque. Bajada Pucará 1900. Código Postal. 5000. Córdoba Capital (Argentina) 


\section{Introducción y objetivos}

Se consideran hernias inguinoescrotales gigantes a aquellas que se extienden por debajo del punto medio de la cara interna del muslo, con el paciente en posición de pie (1).

La reparación libre de tensión fue descrita por Lichtenstein en 1987, y es considerada por muchos como el gold standard en el manejo quirúrgico de la cirugía de la hernia inguinal (2).

Se define técnicamente como hernia o eventración abdominal con pérdida de domicilio, cuando más del $50 \%$ del contenido de la cavidad abdominal se encuentra fuera de la misma (3). Estas grandes hernias plantean un problema significativo, debido al compromiso cardiorrespiratorio siguiente al incremento súbito de la presión intraabdominal, luego de la reposición del contenido visceral. Para evitar estos inconvenientes, numerosas técnicas fueron propuestas, entre ellas el neumoperitoneo progresivo de Goñi Moreno (4).

El objetivo de este trabajo es presentar el caso inusual de una hernia inguinoescrotal gigante con pérdida de domicilio, tratada con neumoperitoneo progresivo preoperatorio (NPP) y posterior reparación del defecto abdominal con una hernioplastia libre de tensión con malla, según la técnica propuesta por Lichtenstein.

\section{Caso clínico}

Paciente de sexo masculino de 50 años de edad, con antecedentes personales de sedentarismo, hipertensión arterial, tabaquismo y obesidad mórbida (IMC 46), consultó por presentar una hernia inguinoescrotal derecha de 15 años de evolución. Al examen físico presentaba una tumoración inguinal de $70 \times 30 \mathrm{~cm}$ irreductible manualmente, acompañada de trastornos tróficos (induración escrotal y lesión ulcerosa) (fig. 1).

La tomografía abdominal y pélvica con contraste endovenoso informó un voluminoso saco herniario con contenido intestinal y omental, quedando el compartimento abdominal prácticamente vacío (fig. 2).

Al inicio de la internación se colocó un catéter en fosa ilíaca izquierda según técnica de Seldinger, para la realización de N.P.P. En un período de 20 días se insuflaron diariamente entre 600 y $800 \mathrm{ml}$ de aire hasta un volumen total de $16600 \mathrm{ml}$ (fig. 3). Se realizaron tres controles ventilatorios por espirometría, demostrando restricción respiratoria leve.

Durante la cirugía se efectuó un abordaje inguinal derecho a través de una incisión de Gregoire, identificándose un saco herniario directo el cual contenía asas de intestino delgado, colon ascendente no deslizado y su respectivo epiplón mayor (fig. 4). El contenido herniario fue reducido y posteriormente el defecto anatómico fue reparado con la colocación de una malla de polipropileno de $20 \times 15 \mathrm{~cm}$, según técnica de Lichtenstein (fig. 5). Se resecó el escroto remanente y un drenaje hemosuctor fue colocado antes del cierre de la herida.

El paciente fue admitido en la unidad de cuidados intensivos luego de la cirugía para observación durante 1 día. Posteriormente pasó a sala general y fue dado de alta al cuarto día. Los controles inmediatos y alejados hasta los 12 meses demostraron una buena recuperación psicofísica, y sin recurrencia (fig. 6).

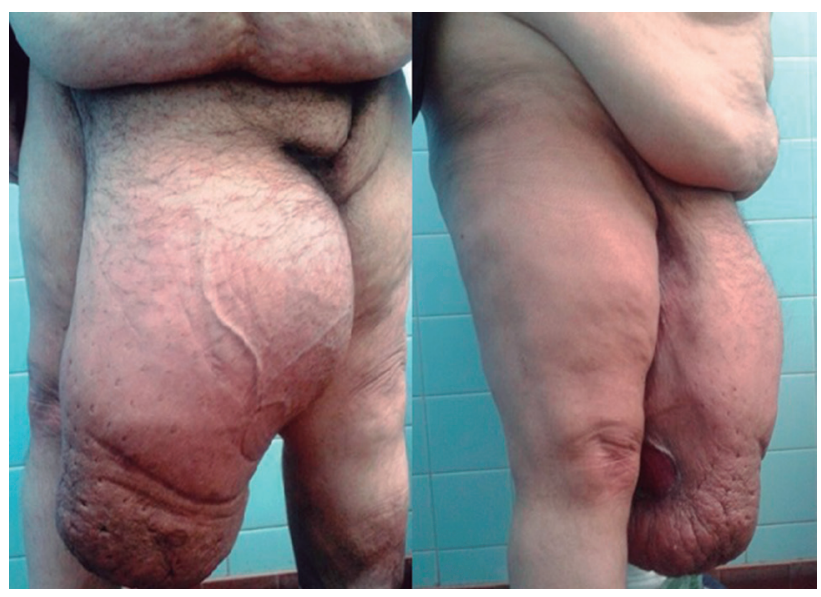

Figura 1. Hernia inguinoescrotal derecha gigante.

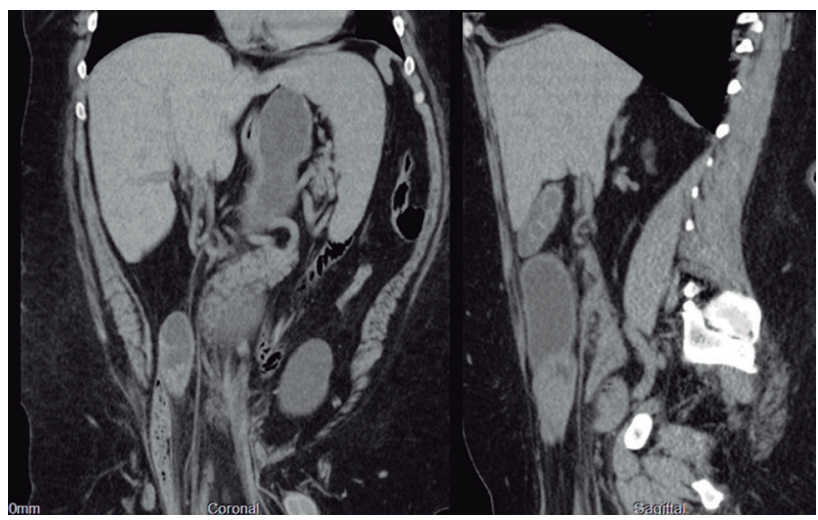

Figura 2. Tomografía abdominal y pélvica con contraste endovenoso.

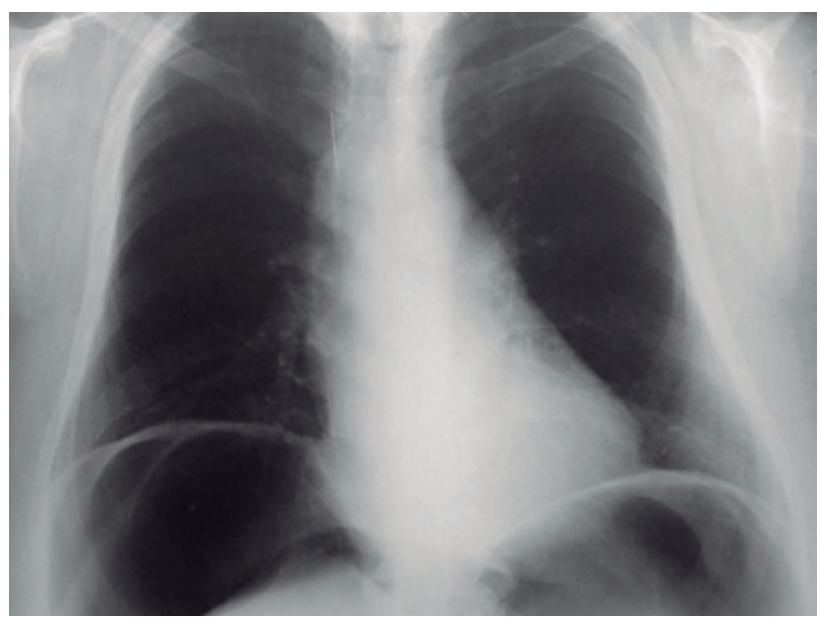

Figura 3. Radiografía de tórax donde se observa el neumoperitoneo preoperatorio. 


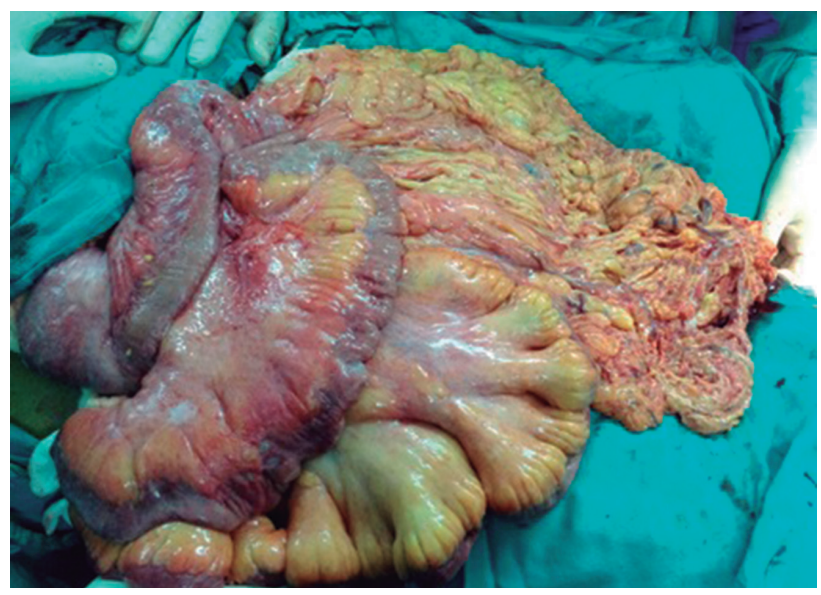

Figura 4. Contenido herniario.

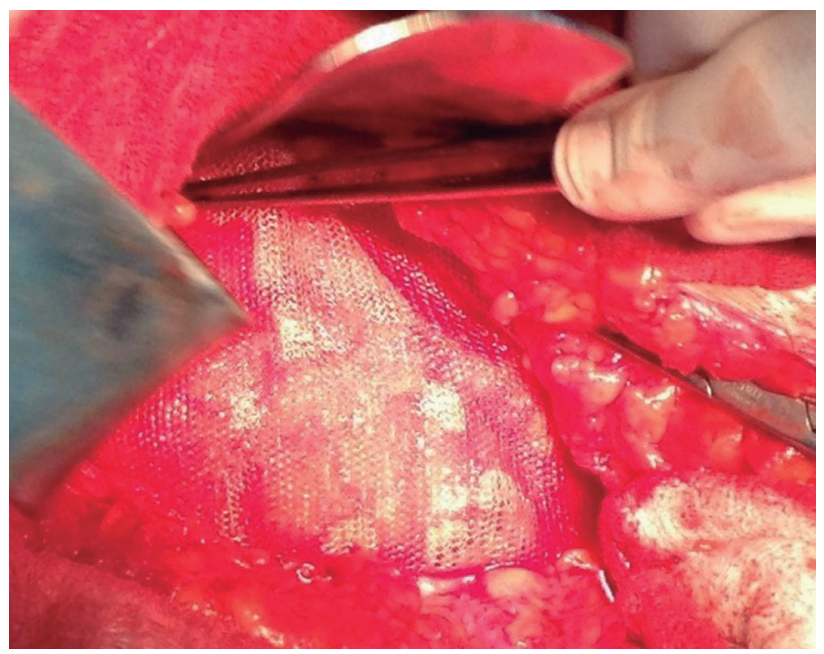

Figura 5. Colocación de malla de polipropileno.

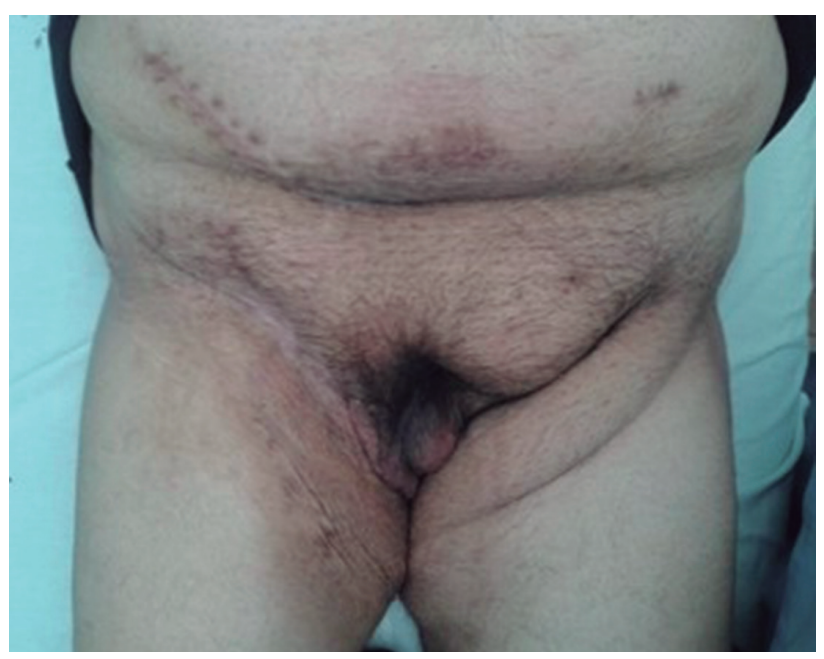

Figura 6. Control a los 12 meses de la reparación.

\section{Discusión}

Las hernias inguinoescrotales son poco frecuentes en los países desarrollados y, usualmente, son solo vistas en la práctica médica cuando el paciente se presenta luego de varios años de negligencia. La pérdida de domicilio ocurre porque la cavidad abdominal se ha adaptado a encontrarse vacía por un largo período de tiempo. Esto genera una disminución de la presión intraabdominal (PIA) que conlleva a un acortamiento progresivo de los músculos de la pared abdominal y una reducción de la capacidad abdominal. A nivel intestinal, por compresión del anillo herniario, se produce una disminución en el retorno venoso y linfático con el consecuente edema de las asas intestinales. Por último, a nivel pulmonar, el hemidiafragma izquierdo desciende, lo que genera un trastorno ventilatorio restrictivo (5-7).

Los síntomas y signos acompañantes a estas hernias son: dolor abdominal crónico, dificultad para desplazarse y efectuar los quehaceres de la vida cotidiana, dolor de espalda, dificultad en la función sexual, insuficiencia respiratoria, obesidad o extrema delgadez, erosión de la piel en el área de la hernia o en áreas de pliegues cutáneos, adherencias de asas intestinales o fístulas enterocutáneas o enteroenterales (8). Estos problemas perjudican severamente la calidad de vida del paciente, con un considerable impacto social y psicológico.

Reducir el gran contenido herniario al abdomen podría causar un síndrome compartimental, en el cual el diafragma es empujado hacia la cavidad torácica generando dificultad respiratoria y compromiso en el retorno venoso, lo que puede ocasionar un colapso circulatorio (9). Además, la reintroducción forzada del intestino al abdomen puede producir obstrucción intestinal, dehiscencia de la herida y recurrencia.

Para evitar este problema y combatir la pérdida de domicilio, varias técnicas adyuvantes a la cirugía fueron descritas (10): por un lado, se encuentran las que se llevan a cabo en la fase preoperatoria (prequirúrgicas) y, por el otro, aquellas realizadas en forma intraoperatoria (transquirúrgicas).

Dentro de las técnicas prequirúrgicas encontramos la colocación de expansores tisulares, la aplicación de toxina botulínica y la confección de NPP. Los expansores tisulares, son implantes de silicona colocados entre los músculos oblicuos mayor y menor que, elongando la pared lateral del abdomen, permiten el cierre posterior del defecto (11). La aplicación de la toxina botulínica A en los músculos abdominales, es un procedimiento simple y ambulatorio que provoca una parálisis de los mismos, disminuyendo su espesor y aumentando su longitud y el diámetro transverso abdominal (12).

Nuestra hernia, acorde a la clasificación de Trakarnsagna et al. (13) fue tipo III, por lo que optamos realizar un incremento del volumen intraabdominal a través del NPP. Existen varios beneficios con respecto al mismo. Por un lado, el aire insuflado causa un aumento de la PIA y del volumen de la cavidad abdominal, producto de la relajación y elongación muscular. Esto genera una disminución de sangre venosa en la circulación esplénica; por ende, el líquido intersticial en los tejidos intraabdominales disminuye, incluidas las asas intestinales. Es así que el NPP no solo aumentaría el continente, sino que también disminuiría el contenido (14). Por otro lado, produce una adherenciolisis, diseca el saco herniario y permite localizar otras hernias o debilidades 
de la pared que no han sido previamente evidenciadas. Además, el estiramiento de la piel escrotal ayuda en la limpieza cutánea y puede potencialmente disminuir la incidencia de infecciones (15). Las limitaciones de esta técnica son la hospitalización prolongada y la difusión del aire al saco herniario.

Por último, las técnicas transquirúrgicas tienen el objetivo de aumentar el volumen de la cavidad mediante la separación de componente (16) o por incisiones descarga (17). Otras, disminuyen quirúrgicamente el contenido herniario (debulking) (18) a través de la resección del colon, intestino delgado o del omento mayor. Sin embargo, estas fueron asociadas a un gran riesgo de infección protésica o de dehiscencia de la anastomosis (13).

\section{Conclusión}

El neumoperitoneo progresivo es un método seguro y fácil de realizar, previo al tratamiento quirúrgico de las grandes hernias inguinoescrotales que han perdido el domicilio. No solo permite una expansión de la cavidad abdominal, lo que disminuye el riesgo de un síndrome compartimental posquirúrgico, sino que, además, efectúa una disección neumática del saco herniario y de su contenido, facilitando su reducción durante el acto operatorio.

\section{Bibliografía}

1. Hodgkinson DJ, Mc Ilrath DC. Scrotal reconstruction for giant inguinal hernias. Surg Clin N Am. 1984;64:307-12.

2. Lichtenstein IL, Shulman AG, Amid PK, Montlor MM. The tension-free hernioplasty. Am J Surg. 1989;157:188-93.

3. López Sanclemente MC, Robres J, López Cano M, Barri J, Lozoya R, López S, et al. Neumoperitoneo preoperatorio progresivo en pacientes con hernias gigantes de la pared abdominal. Cir Esp. 2013;91(7):444-9.

4. Goñi-Moreno I. Chronic eventrations and large hernias; preoperative treatment by progressive pneumoperitoneum- original procedure. Surgery. 1947;22:945-53.
5. Correa R, Canals PP, Mederos O, Roselló J, Esperón R, Mustafá L. Reparación de hernias incisionales grandes con mallasupraaponeurótica. Rev Cubana Cir. 2002;41:156-63.

6. Mayagoitia JC, Suárez D, Arenas JC, Días de León V. Preoperative progressive pneumoperitoneum in patients withabdominal-wall hernias. Hernia. 2006;10:213-7.

7. Willis S, Schumpelick V. Use of pneumoperitoneum in the repair of gigant hernias. Hernia. 2000;4:105-11x.

8. Reconstrucción de las hernias complejas de pared abdominal anterior: un problema de enfoque multidisciplinario. Rev Hispanoam Hernia. 2015;3(2):47-8.

9. Serpel JW, Polglase AL, Anstee EJ. Giant inguinal hernia. ANZ J Surg. 1988;58:831-4

10. Ibarra Hurtado TR, Negrete Ramos GI, Preciado Hernandez F, Nuño Guzmán CM, Tapia Alcalá E, Bravo Cuellar L. Toxina botulínica A como adyuvante en hernia inguinoescrotal bilateral con pérdida de domicilio. Informe del primer caso y revisión de la bibliografía. Rev Hispanoam Hernia. 2014;2(4):139-44.

11. Jacobsen AM, Petty P, Bite U. Massive abdominal-wall hernia reconstruction with expanded external/internal oblique and transversalis musculofascia. Plast Reconstr Surg. 1997;100:326-35.

12. Ibarra TR, Nuño CR, Echeagaray JE, Robles E, González JJ. Use of botulinum toxin type A before abdominal wall hernia reconstruction. World J Surg. 2009;33:2553-6.

13. Trakarnsagna A, Chiswangwatanakul V, Methasate A, Swangsri J, Phalanusitthepha C, Parakonthun T, et al. Giant inguinal hernia: Report of a case and reviews of surgical techniques. Int J Surg Case Rep. 2014:868-72.

14. Rappoport Stramwasser J, Carrasco Toledo J, Silva Solís JJ, Albán García M, Papic Silva F. Neumoperitoneo terapéutico preoperatorio en el tratamiento de la hernia incisional gigante. Reducción del volumen visceral como explicación fisiopatológica de sus beneficios. Rev Hispanoam Hernia. 2014;2(2):41-7.

15. Cavalli M, Biondi A, Bruni PG, Campanelli G. Giant inguinal hernia: the challenging hug technique. Hernia. 2015;19:775-83.

16. Ramírez OM , Ruas E, Dellon L. Components separation» method for closure of abdominal-wall defects: An anatomic and clinical study. Plast Reconstr Surg. 1990;86:519-26.

17. Carbonell-Tatay F, Moreno-Egea A, editores. Eventraciones. Otras hernias de pared y cavidad abdominal. Picanya: Ed. Vimar; 2012.

18. El Saadi AS, Wadan AH, Hamerna S. Approach to a giant inguinoscrotal hernia. Hernia. 2005;9:277-9. 